\title{
Three-Dimensional Road Network by Fusion of Polarimetric and Interferometric SAR Data
}

\author{
P. Gamba ${ }^{(*)}$, B. Houshmand ${ }^{(\dagger)}$ \\ (*)Dipartimento di Elettronica, Università di Pavia, Via Ferrata, 1, I-27100 Pavia, Italy \\ (†)Jet Propulsion Laboratory, 4800 Oak Grove Drive, Pasadena, CA 91109-8099, USA
}

\begin{abstract}
In this paper a fuzzy classification procedure is applied to polarimetric radar measurements, and street pixels are detected. These data are successively grouped into consistent roads by means of a dynamic programming approach based on the fuzzy membership function values. Further fusion of the $2 \mathrm{D}$ road network extracted and 3D TOPSAR measurements provides a powerful way to analyze urban infrastructures.
\end{abstract}

\section{INTRODUCTION}

The challenge of remote sensing over urban environments is mainly to find the way to merge the information available at different scales with different sensors and retrieve thematic or, better, detailed maps of the structures and infrastructures. To this aim, very promising results have been reported by the fusion of interferometric SAR and AVIRIS data over Los Angeles [1]. In this paper, instead, we want to focus on the synergies of polarimetric and interferometric SAR measurements in the extraction of the road network over the same area.

It is quite well assessed that polarimetric SAR images are very useful in detecting streets and roads, due to their strong interaction with the radar pulse, caused by their orientation with respect to the SAR sensor [2]. This makes the polarimetric images of a densely urbanized area the best means to detect the zones where these infrastructures are located. However, a simple classification is not able to group consistent sets of pixels in street segments. Therefore, to the classification step must follow a suitable pixel grouping procedure, as already noted in [3]. To this aim, in that paper a modified fuzzy Hough transform was introduced, with streets extracted always as straight lines.

In this paper, we make a first comparison of this approach and a dynamic programming method for road extraction [4]. First, the SAR data is analyzed by means of fuzzy clustering, and road pixels are individuated. Then, these pixels are grouped looking recursively for the shortest path in the image and discarding already considered pixels. These paths are dynamically extracted by following curved lines prized with their roughness. In this way, the locally irregular behavior of the street pixels, due to errors in the classification step are smoothed by the regularization introduced by the proposed methodology.

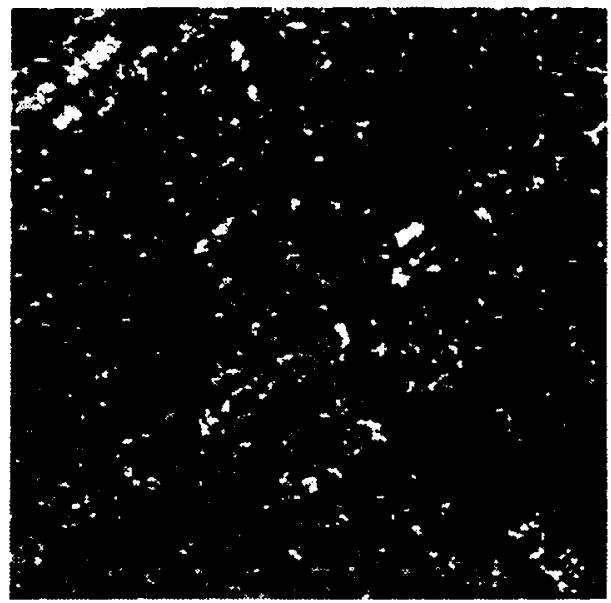

Figure 1: A VV polarization AIRSAR image over Los Angeles.

Finally, the results of the preceding step are used to mask interferometric SAR data to extract the 3D structure of the complete road network.

\section{ROAD EXTRACTION VIA FUZZY CLUSTERING AND DYNAMIC PROGRAMMING}

As already outlined in [3], fuzzy classification methods, like Fuzzy-C-Means (FCM) and Possibility-C-Means (PCM) [5] are extremely suitable for the extraction of the most relevant urban features from SAR data. The same approach is presented in this work, where polarimetric SAR images over a urban environment are classified by means of these approaches and then, before all the fuzzy membership values are lost with the final classification step, all those pixels that belong to the street class are possibly grouped into consistent lines, corresponding to roads.

\subsection{Iterative regularized shortest-path extraction}

The basic dynamic programming algorithm follows the idea to find the path with the lowest cost to go from one side of an image to the other, where this cost is computed by means of the sum of the grey levels of the path pixels [4]. A regularization term is added, in order to take 


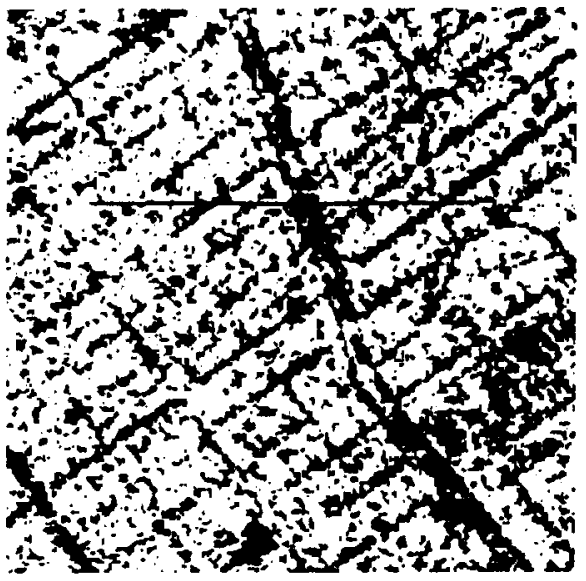

Figure 2: Fuzzy street classification results after applying the FCM algorithm the VV polarization and magnitude images recorded over Los Angeles.

into account the curvature of the line, and prize smoother sections. Moreover, in order to avoid the possibility to find only straight lines connected by large bends, a pixel subdivision is considered. In other words, a top-bottom path is searched in the original image so that

$$
J=\sum_{r=1}^{m} X\left(r, j_{r}\right)+\lambda \sum_{r=2}^{m-1}\left(j_{r-1}-2 j_{r}+j_{r+1}\right)^{2}
$$

is minimized, after subdividing horizontally each pixel into $q$ sub-pixels. In this formula $m$ is the row number, $X(i, j)$ is the grey level value at the $(i, j)$ location, and $\lambda$ is a weighting constant.

In our procedure the same approach is implemented, starting from a grey-level image that is the normalized version of the classified SAR image. To all the pixels with street membership function less than a given threshold $t$ a value of 255 is assigned. Instead, street pixels are translated into a grey level image where $X(i, j)$ is a suitably stretched version of the membership values. Moreover, since more than one road must be extracted from the original image, the cost function is defined for all the border pixels, and computed by suitably exchanging the indexes in eq.(1). In other words, again for a top-bottom path, the cost function translates into

$$
J(j)=\sum_{r=1}^{m} \bar{\mu}_{r j_{r}}^{s}+\lambda \sum_{r=2}^{m-1}\left(j_{r-1}-2 j_{r}+j_{r+1}\right)^{2}
$$

with the constraint that $j_{1}=j, \mu_{i j}^{*}$ is equal to the membership values of the $(i, j)$-th pixel of the street class, and

$$
\bar{\mu}_{i j}^{s}=\left(1-\frac{\mu_{i j}^{s}-t}{1-t}\right) 255
$$

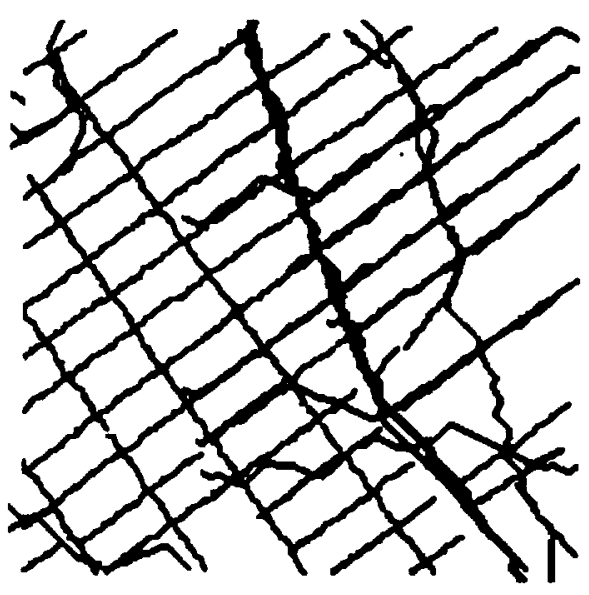

Figure 3: Some of the roads extracted starting from the pixels belonging to the street class of the classification in fig. 2, with superimposed the ground truth.

Therefore, first a gray level image is determined after the FCM/PCM classification step. Then, starting from a randomly chosen boundary point, the shortest path to the other side of the image is searched, and all the points belonging to the road extracted and to its neighborhood (up to a 2 pixel distance) are discarded. Successively, a new random boundary point (with the only constraint to be sufficiently far from the previous one) is chosen, and an other path is determined. Iteratively, this procedure restores the road network, even if street crosses are cancelled from the image as soon as a single road passing through them is found.

To introduce roads ending inside the image, we limit the number of white (high prize) pixels that we may add to the path to push it longer and look for successive street pixels. Of course, this value is chosen so that the algorithm is still able to fill simple line gaps.

\section{EXPERIMENTAL RESULTS}

The outlined procedure was applied to AIRSAR/TOPSAR data recorded over Los Angeles. In particular, the Lband AIRSAR images recording the VV polarization and the complete magnitude of the backscattered field were used as input of the FCM algorithm. The VV polarization image is shown in fig. 1 , while in fig. 2 the fuzzy street classification results (three classes were considered: street, buildings and green areas) are presented, with defuzzyfication threshold $t$ equal to 0.5 . Finally, some of the roads extracted by means of the dynamic programming approach are depicted in fig. 3, superimposed on the ground truth, i. e. the road network manually determined from the same aerial images.

Fig. 4, instead, presents the road network extracted by means of the pyramidal Fuzzy Connectivity Hough Trans- 


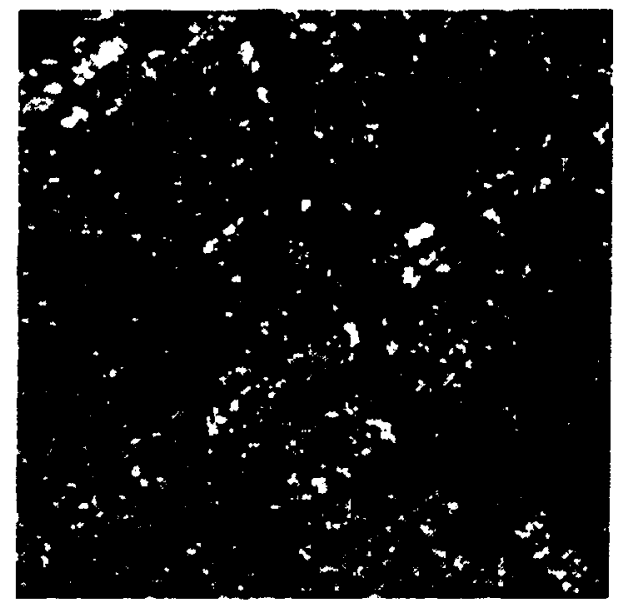

Figure 4: The same road network of fig. 3 extracted, after the fuzzy classification, by means of the Fuzzy Connectivity Hough Transform.

form. We may observe that a largest number of pixels are clustered in road, but all of them are straight lines, and erroneously classified pixels are also more than in fig. 3 . On the other hand, connectivity assures a better recognition of curve segments starting and/or ending inside the image.

\subsection{Fusion of SAR and IFSAR data}

The roads found by the above presented algorithm were used to mask the 3D Digital Surface Model (DSM) extracted from Interferometric SAR (IFSAR) measurements. Indeed, the same airplane carrying the AIRSAR sensor carries also the TOPSAR sensor, able to retrieve the surface 3D topology by means of computing the difference in phase of the radar responses recorded by two near antennas.

An example of the results achieve is. shown in fig. 4, where the $3 \mathrm{D}$ road network of a small portion of Los Angeles is presented. It is straightforward that such a representation allows to

- refine the classification of 2D polarimetric images by discarding points recorded as roads but clearly outside the road level;

- compute $3 \mathrm{D}$ information about roads (like the slope) that may be useful for urban planning, disaster management, environmental studies,

\section{CONCLUSIONS}

This work shows that a suitable fusion of data available by SAR sensors may provide a lot of useful information about urban environments. In particular, we focused on

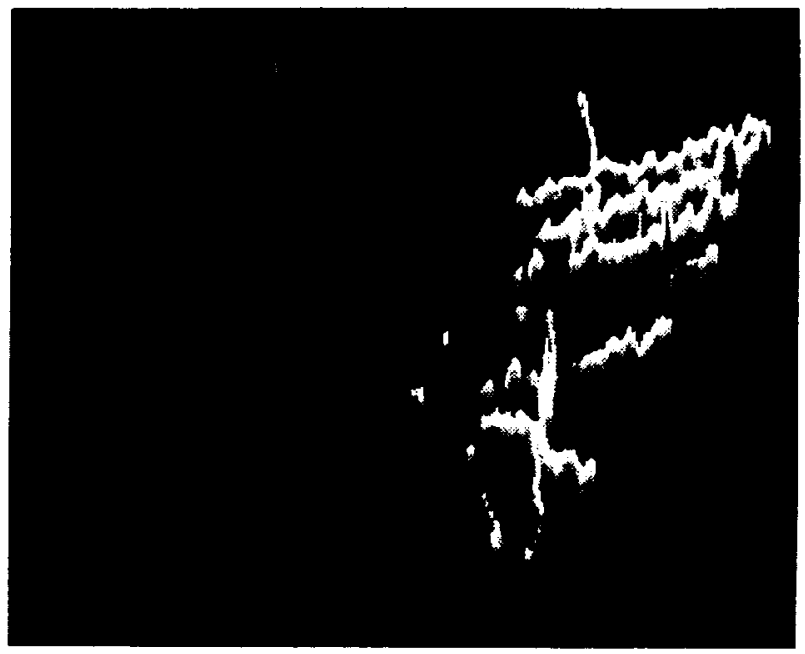

Figure 5: A perspective image of the 3D road network obtained by fusion of the roads shown in fig. 3 and the coregistered digital surface obtained by IFSAR measurements.

the road extraction problem, and showed that $2 \mathrm{D}$ and $3 \mathrm{D}$ analysis of the same location can be merged to retrieve the $3 \mathrm{D}$ road network structure with a certain degree of accuracy.

This representation allows to consider, for instance, the problems due to hydrological hazards transforming this network into a rain or water collector, as well as to identify automatically dangerous points of the same road system.

\section{REFERENCES}

[1] G.F. Hepner, B. Houshmand, I. Kulikov, and N. Bryant, "Investigation of the potential for the integration of AVIRIS and IFSAR for urban analysis," Photogrammetric Eng. Remote Sensing. Vol. 64, No. 8, pp. 512-520,1998.

[2] C.J. Oliver, "Information from SAR images," $J$. of Physics D, Applied Physics, Vol. 24, n. 9, pp. 14931514, 1991.

[3] P. Gamba and P. Savazzi, "Classification of urban environments in SAR images: a fuzzy clustering perspective," Proc. of the 1998 IEEE International Geoscience and Remote Sensing Symposium (IGARSS'98), Vol. I, pp. 351-353, Seattle, WA, July 1998.

[4] M. Buckley and J. Yang, "Regularised shortest-path extraction," Pattern Recognition Lett., Vol. 18, pp. $621-62,1997$

[5] R.N. Dave' and R. Krishnapuram, "Robust clustering methods: a unified view," IEEE Trans. on Fuzzy Systems, Vol. 5, No. 2, pp. 270-293, May 1997. 